\title{
Simulation and Implementation of an Improved Inscribed Chord Interpolation Algorithm
}

\author{
Hui Zhang, Qinruo Wang \\ School of Automation, Guangdong University of Technology, Guangzhou, 510006, China \\ E-mail: zh2008@gdut.edu.cn
}

\begin{abstract}
In order to improve the shortcomings of the realization of the traditional quadratic curve interpolation inscribed chord method, this paper proposes an improved internal chord interpolation algorithm. The program flow chart of the algorithm achievement and the interpolation method of circular arc interpolation and linear interpolation of a detailed operation process is given in this paper. Finally through a calculation example the simulation is conducted, and the point by point comparison method were compared. The simulation results show the improvement on the quadratic curve interpolation in the inner string interpolation speed, good flexibility, and it worth popularizing in the interpolation algorithm for real time interpolation.
\end{abstract}

Keywords- quadratic curve interpolation; improved chord line interpolation; point by point comparison method; the study on simulation

\section{INTRODUCTION}

Interpolation is an extremely important feature of the entire computer numerical control system. The traditional point by point comparison method is mainly used for the earlier hard-line CNC systems, and stepper motor is used to drive generally[1]. The implementation method of this algorithm is relatively simple, which usually only through the addition and shift operations can complete the interpolation and it has been widely used in graphics devices and CNC machine tools. Now the NC materials still spend a lot of chapters on this algorithm because understand the algorithm is beneficial to learn other more complex interpolation algorithm[2]. However, the interpolation algorithm is gradually replaced by other algorithms due to slow velocity of interpolation and poor straight smoothing itself. Whether foreign or domestic, high precision, high speed and new interpolation algorithm (software) have been efforts to break through the difficulties of the researchers, but also the confidential technical core that various CNC companies try their best to protect. Such as Siemens, Fanuc CNC system, many of its features are open to the users, but the interpolation software never open to their users[3].

The inscribed chord method is the one that using the adjacent chord between the two sampling points in the curve to approximate the corresponding curve[4-5]. And the interpolation points must satisfy two conditions, one is that the interpolation point should be located on the interpolation curve, the coordinates of the point should meet the curve equation, another is that the distance between the current interpolation point with a previous one should meet the feed rate requirements. One equation group that consist of two equations can be obtained according to these two conditions. Solving this equation group, and based on a given approximation error, the coordinates of the interpolation point can be got. Generally speaking, the equation group is more complex as to a general quadratic curve, and only iterative method for solving the calculation, which is timeconsuming, and not suitable for real-time interpolation[6].

Another idea of a transformation that is calculated by the method of curve intersection interpolation points, the current interpolation point is the cross point belonging to the interpolated curve and the circle whose center and radius is the previous interpolation point and the interpolation segment length[8-10]. But there is not much researchers doing their work in this method. In this case, this paper put forward the error judgment that base on the method which mentioned above. Simulate on MATLAB and the results show that the new method has improved interpolation speed and has a better flexibility.

\section{IMPROVED INSCRIBED CHORD INTERPOLATION}

The improved internal chord interpolation method is shown as follows in Fig.1, take a certain point of the trajectory of the ideal interpolation as a starting interpolation point, and decide the end point. Consider the starting interpolation point as the center, the interpolation segment length $r$ is the radius of the circle intersect with the ideal interpolation trajectories. The intersection obtained above, which is the next interpolation point. Detect whether the intersection is within the allowable error range or not. If it does, take the intersection as the center of the circle, and the interpolation line segment length of radius $r$ round continues with the ideal interpolation trajectories intersect; If it does not, then this intersection, the ideal interpolation point on the trajectory of the center, the interpolation segment length $r$, which is the radius of the circle, continue intersects with the ideal curve at the same time, detecting whether to arrive the end of this cycle, obtaining all interpolation points. 


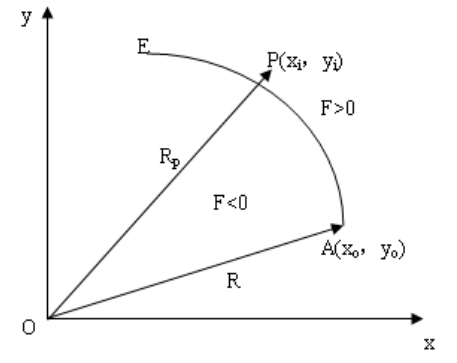

Figure 1. Inscribed chord interpolation

The working process of improved inscribed string interpolation method needs to pass to five steps which are circle center determination, curve intersection, error judgment, coordinate feed, end judgment. And its working process is shown in fig.2.

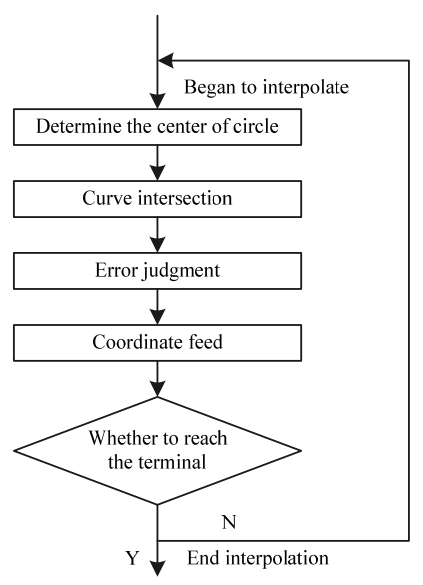

Figure 2. Working process of the interpolation

According to the working process of the interpolation, the flow chart of improved inscribed chord interpolation calculation program is shown in Fig. 3.

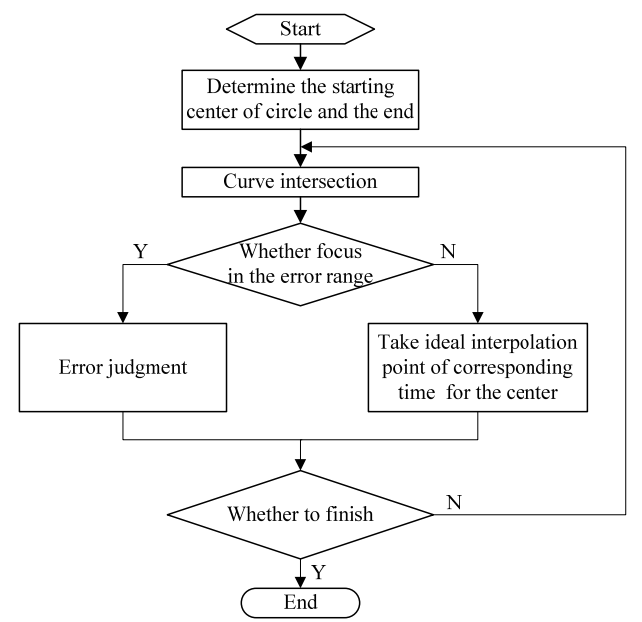

Figure 3. Flow chart of improved inscribed chord interpolation calculation program

\section{IMPROVED INSCRIBED CHORD CIRCULAR ARC INTERPOLATION}

The simulation uses point by point comparison method for linear interpolation and point by point comparison method for circular arc interpolation as reference, interpolates the line $\mathrm{OA}$ and the arc $\mathrm{AB}$ respectively, programs and simulates on MATLAB. The step length is one in the new interpolation method.

\section{A. Linear interpolation}

Assume the line OA is the one processed, the starting point is at origin, the coordinate of the end, $x_{e}=4, y_{e}=3$, so the judgment value of end can be set as this: $E_{7}=x_{e}+y_{e}=7$. At the beginning, the deviation is 0 . According to the Point by point comparison Algorithm, the interpolation trajectory of the line OA is shown in Fig. 4.

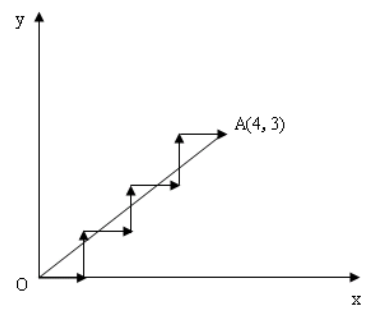

Figure 4. Point by point comparison method for linear interpolation trajectory

For the same processed line OA, improving method for inscribed chord interpolation can be used. The starting point of the coordinate is $(0,0)$, the end point is $(4,3)$. According to the discrimination of the end, the error range is $0.5 \%$. The process of improving method for inscribed chord interpolation is listed in Table I.

TABLE I. THE PROCESS OF IMPROVING METHOD FOR INSCRIBED CHORD INTERPOLATION

\begin{tabular}{|c|c|c|c|c|}
\hline $\begin{array}{l}\text { Step } \\
\text { No. }\end{array}$ & $\begin{array}{l}\text { Center of } \\
\text { the Circle }\end{array}$ & $\begin{array}{c}\text { Point of } \\
\text { Intersection }\end{array}$ & $\begin{array}{c}\text { Error } \\
\text { Judgment }\end{array}$ & $\begin{array}{c}\text { End } \\
\text { Judgment }\end{array}$ \\
\hline & & & $\begin{array}{c}\text { Whether in } \\
\text { the error range }\end{array}$ & $\begin{array}{l}\text { Whether to } \\
\text { reach the end }\end{array}$ \\
\hline 1 & $(0,0)$ & $(0.8,0.6)$ & Yes & No \\
\hline 2 & $(0.8,0.6)$ & $(1.6,1.2)$ & Yes & No \\
\hline 3 & $(1.6,1.2)$ & $(2.4,1.8)$ & Yes & No \\
\hline 4 & $(2.4,1.8)$ & $(3.2,2.4)$ & Yes & No \\
\hline 5 & $(3.2,2.4)$ & $(4.0,3.0)$ & Yes & No \\
\hline
\end{tabular}

According to the operation of Table I, the trajectory of improving method for inscribed chord linear interpolation is shown in Fig.5. 


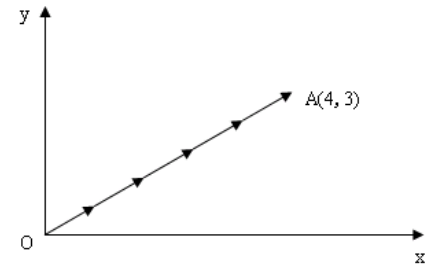

Figure 5. Improved inscribed chord linear interpolation trajectory

\section{B. Circular Arc Interpolation}

Assume that the anticlockwise arc $\widehat{A B}$ wanted to process is in the first quadrant. The coordinate of the starting point $\mathrm{A}$ is $(4,0)$, the coordinate of the end is $(0,4)$, the judgment value of the end, $\mathrm{E}=\left|\mathrm{x}_{\mathrm{i}}-\mathrm{x}_{0}\right|+\left|\mathrm{y}_{\mathrm{i}}-\mathrm{y}_{\mathrm{o}}\right|=8$. According to the Point by point comparison Algorithm, the interpolation trajectory of the arc $\widehat{\mathrm{AB}}$ is shown in Fig. 6 .

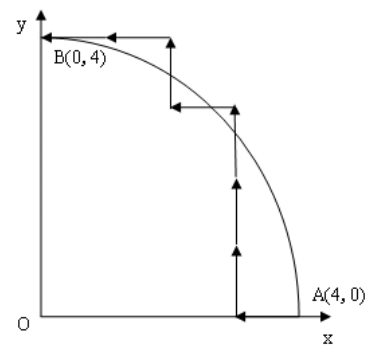

Figure 6. Circular arc interpolation trajectory of point by point comparison method

For the same circular arc $\overparen{A B}$ processed, improving method for inscribed chord interpolation is used. Namely the starting point of the coordinate is $(4,0)$, the end point is $(0$, 4). According to the finishing point judgment, the error range is $0.5 \%$. The new interpolation method of arc interpolation operation process is shown in TABLE II.

TABLE II. THE NEW INTERPOLATION METHOD OF ARC INTERPOLATION OPERATION PROCESS

\begin{tabular}{|c|c|c|c|c|}
\hline $\begin{array}{c}\text { Step } \\
\text { No. }\end{array}$ & $\begin{array}{c}\text { Determine } \\
\text { the Center } \\
\text { of Circle }\end{array}$ & $\begin{array}{c}\text { Intersection } \\
\text { Point }\end{array}$ & $\begin{array}{c}\text { Error } \\
\text { Judgment }\end{array}$ & $\begin{array}{c}\text { Finishing } \\
\text { point } \\
\text { Judgment }\end{array}$ \\
\hline & & & $\begin{array}{c}\text { Whether } \\
\text { to reach } \\
\text { the error } \\
\text { range }\end{array}$ & $\begin{array}{c}\text { Whether } \\
\text { to reach } \\
\text { the end }\end{array}$ \\
\hline 1 & $(4,0)$ & $(3.898,0.894)$ & Yes & No \\
\hline 2 & $(3.898,0.894)$ & $(3.600,1.743)$ & Yes & No \\
\hline 3 & $(3.600,1.743)$ & $(3.119,2.504)$ & Yes & No \\
\hline 4 & $(3.119,2.504)$ & $(2.480,3.138)$ & Yes & No \\
\hline 5 & $(2.480,3.138)$ & $(1.716,3.613)$ & Yes & No \\
\hline 6 & $(1.716,3.613)$ & $(0.864,3.905)$ & Yes & No \\
\hline 7 & $(0.864,3.905)$ & $(-0.030,3.999)$ & Yes & No \\
\hline
\end{tabular}

According to the operation of Table IV, the Circular arc interpolation trajectory of point by point comparison method is shown as Fig. 7.

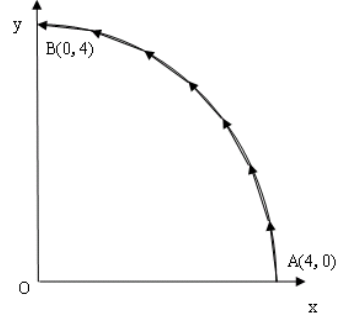

Figure 7. Improved inscribed chord circular arc interpolation trajectory

\section{SyStEM SIMULATION AND RESUltS ANALYSIS}

Through the theoretical analysis and comparison, the improvement of new interpolation method in the speed and accuracy can be seen. Therefore, use MATLAB simulation software to redact algorithm and then simulate. Meanwhile the new interpolation method and point by point comparison method on the same line and circular arc are put into use respectively.

Four groups of simulation test curves are shown in Figure 8 to 11 and experimental results listed in TABLE III.

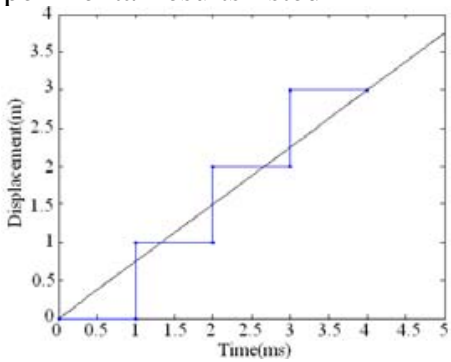

Figure 8. Linear interpolation simulation trajectory of point by point comparison method

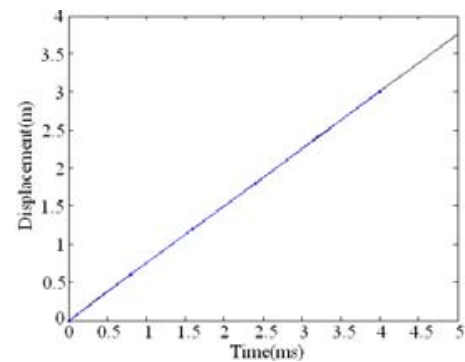

Figure 9. Improved inscribed chord linear interpolation simulation

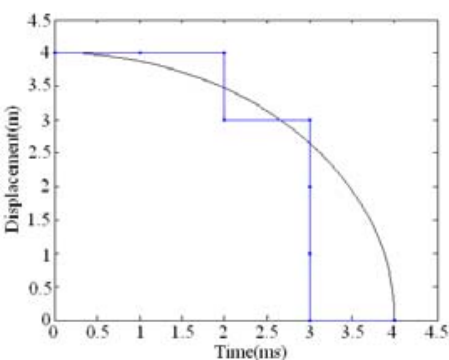

Figure 10. Circular arc interpolation simulation trajectory of point by point comparison method 


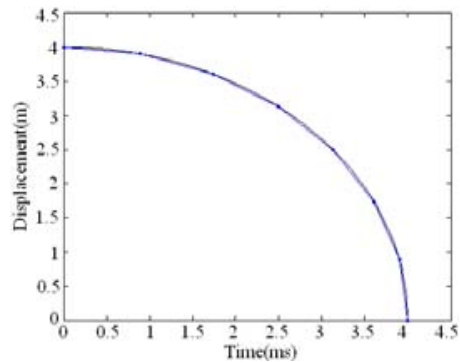

Figure 11. Improved inscribed chord circular arcs interpolation simulation trajectory

According to the simulation trajectory diagrams, the experimental results of two kinds of interpolation algorithms are listed in TABLE III. Improved inscribed chord interpolation algorithm has moderate improvement in interpolation speed and accuracy, compared to point by point comparison method, which has certain significance. Nevertheless point by point comparison method is relatively simple, using addition and shift operation method only can complete interpolation usually. But the use of fold line approximating curve, also string included, leading to low precision interpolation and a big approximate error.

TABLE III. COMPARISON OF TWO KINDS OF INTERPOLATION ALGORITHMS SIMULATION RESULTS

\begin{tabular}{|c|c|c|c|c|}
\hline $\begin{array}{c}\text { Interpolation } \\
\text { Method }\end{array}$ & $\begin{array}{c}\text { Point by Point } \\
\text { Comparison } \\
\text { Method for } \\
\text { Linear } \\
\text { Interpolation }\end{array}$ & $\begin{array}{c}\text { Improving } \\
\text { Method for } \\
\text { Inscribed } \\
\text { Chord } \\
\text { Linear } \\
\text { Interpolation }\end{array}$ & $\begin{array}{c}\text { Point by Point } \\
\text { Comparison } \\
\text { Method for } \\
\text { Circular Arc } \\
\text { Interpolation }\end{array}$ & $\begin{array}{c}\text { Method in } \\
\text { This Paper }\end{array}$ \\
\hline Step No. & seven & five & eight & seven \\
\hline $\begin{array}{c}\text { Interpolation } \\
\text { Precision }\end{array}$ & relatively lower & high & $\begin{array}{c}\text { relatively } \\
\text { lower }\end{array}$ & $\begin{array}{c}\text { relatively } \\
\text { higher }\end{array}$ \\
\hline
\end{tabular}

In addition, discriminant derivation of point by point comparison method is based on quadrant division. Therefore, each quadrant have their own discriminant and different traveling direction, which brings a lot of inconvenience to the interpolation operation. Namely before the interpolation, judge the quadrant in which the segment stays according to the trend of the segment, and then choose the different interpolation formulas to carry out operation. However, the improved inscribed chord interpolation method, which is a kind of unified algorithm, simplifying the algorithm program and improving interpolation speed without considering quadrant relationships.

During the working process of high-speed CNC punch press, oscillation and external factors happened inevitably. While in the interpolation process, small oscillation can make the interpolation points deviate, thus affecting the entire interpolation results and making product quality poor. The new interpolation method with error detection added, automatically choose the point qualified as new interpolation points, on condition that the deviation error of the interpolation points is in the allowable ranges due to factors such as machine shock deviation error bounds, thus providing more security in the precision.

\section{CONCLUSIONS}

This paper mainly studies the concrete realization of the internal chord interpolation algorithm. A new interpolation method is proposed on the basis of the study of the traditional interpolation methods, ideas and feasibility of the new interpolation method are analyzed. Circular arc interpolation and linear interpolation are realized through MATLAB simulation, also with the traditional point by point comparison method compared. The simulation results show that the improved inscribed chord interpolation speed of quadratic curve interpolation is fast and has good flexibility itself, which is a interpolation algorithm worthwhile realtime interpolation operation. Of course, in the modern manufacturing industry, the surface processing and quality requirements continue to increase, leading a higher and higher interpolation speed. But speed and accuracy are two mutual restriction factors, interpolation accuracy decrease corresponding to the interpolation speed increase on the circumstance that interpolation frequency is limited by hardware conditions. Therefore, pretreatment or real-time prospective method can be used to simplify the interpolation algorithm, thus speeding up the interpolation speed, which is the further research work needs to be done.

\section{REFERENCES}

[1] Kim D.I, "Study on interpolation algorithms of CNC machine tools", IEEE Industry Applications Conference, vol. 3, 1995, pp. 1930-1937.

[2] Q.M.Ni, S.L.Wang, C.X.Li, and X.Y.Yuan, "Surface interpolation technique in CNC Machine”, Mechatronics, vol. 6, pp. 34-36,2000.

[3] X.H. Mao, "Improving method for straight line function circular arcs interpolation" , Journal of Henan Institute of Science and Technology, vol. 34, pp. 95-97, 2006.

[4] Y.H.Wang, "Research on minimum deviation interpolation algorithmic optimization in CNC system", Journal of Qiqihar University, vol. 24, pp. 79-82, 2008.

[5] B. Xie, "Optimal design on interpolation algorithm of numerical control system”, Journal of Anhui Vocational Technical College, vol. 5, pp. 1-4, 2006.

[6] L.J. Chen, "Method of 5-axis Spline Interpolation and Realizetion in CNC System”, Proc. International Asia Conference on Informatics in CAR, 2009, pp. 398-401.

[7] M.H. Xie, L. Ye, "Research on high speed interpolation algorithm of NURBS curve for computer numerical control machine”, Joural of Hennan Polytechinc University ( Natural Science), vol. 27, pp. 307313,327, 2008.

[8] S.Y. Li, W. Luo, "NURBS curve interpolation with high speed and high accuracy \& acceleration-deceleration control method", Computer Integrated Manufacturing Systems, vol. 14, pp. 1142-1147, 2008.

[9] J.W. LI, H. Lin, Y. Sun, "A real time looking-forward NURBS interpolation algorithm”, Machine tools Combination and Automatic Processing Technology, vol. 15, pp. 41-45,49, 2009.

[10] Y.L. Han, J.P. Wang,” Quadratic curve interpolation algorithms”, Joural of the New Technology, vol. 11, pp. 28-30, 2007. 